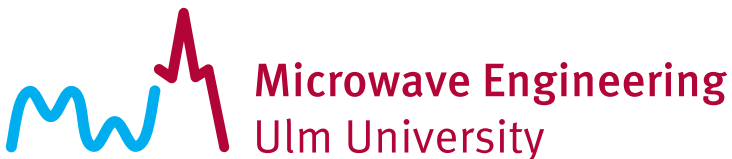

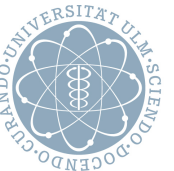

\section{Mechanically Decoupled Transitions from MMIC to Rectangular and Dielectric Waveguides at G-Band}

\author{
Martin Geiger, Martin Hitzler, and Christian Waldschmidt
}

(C) 2020 IEEE. Personal use of this material is permitted. Permission from IEEE must be obtained for all other uses, in any current or future media, including reprinting/republishing this material for advertising or promotional purposes, creating new collective works, for resale or redistribution to servers or lists, or reuse of any copyrighted component of this work in other works. 


\title{
Mechanically Decoupled Transitions from MMIC to Rectangular and Dielectric Waveguides at G-Band
}

\author{
Martin Geiger $^{1 *}$, Martin Hitzler ${ }^{1}$, and Christian Waldschmidt ${ }^{1}$ \\ ${ }^{1}$ Insitute of Microwave Engineering, Ulm University, 89081 Ulm, Germany \\ *martin-2.geiger@uni-ulm.de
}

\begin{abstract}
A variety of transitions from a monolithic microwave integrated circuit (MMIC) to a waveguide were proposed in literature to enable chip-to-chip links or to provide a standard interface. The MMIC is usually in contact with the waveguide and is therefore exposed to the mechanical stress exerted on the overall system. This paper presents mechanically decoupled transitions from MMIC to a rectangular waveguide and to a dielectric waveguide at $\mathrm{G}-\mathrm{Band}(140 \mathrm{GHz}$ to $220 \mathrm{GHz})$. Both transitions use a patch radiator on a quartz glass carrier as on-chip antenna. To focus the beam, a dielectric rod antenna or an elliptical dielectric lens is placed on the quartz glass. Since the transitions are mechanically decoupled, a second antenna is used as receive element. A horn antenna connected to the rectangular waveguide or a second dielectric lens connected to the dielectric waveguide is used. The transitions were characterized using a back end of line MMIC. The minimum measured insertion loss is $3.1 \mathrm{~dB}$ for the transition from MMIC to rectangular waveguide and $4.9 \mathrm{~dB}$ for the transition to dielectric waveguide.

Keywords - MMIC to waveguide transition, MMIC, dielectric waveguide, mechanical decoupling.
\end{abstract}

\section{INTRODUCTION}

The frequency range above $100 \mathrm{GHz}$ has attracted strong interest for high-resolution radars [1], [2] or high-speed communication links [3], [4] due to the large available bandwidth and low cost MMICs. However, the disadvantage of on-chip antennas is the inefficient radiation due to high substrate losses, low substrate heights, and a small chip area. Here, a suitable interface to a rectangular or dielectric waveguide would extend the application spectrum of the MMIC, as for example high gain antennas could be fed. Thus, new applications for radar systems [5] or chip-to-chip communication links [6] would be possible to design.

Several transitions from MMIC to rectangular or to dielectric waveguide were shown in the literature. Low-loss transitions with MMICs completely integrated in the rectangular waveguide were realized with bonds [7] or dipole radiation [8]. Insertion losses (IL) below $1 \mathrm{~dB}$ were achieved. However, the large packaging effort which is required to integrate the MMIC into the waveguide with its DC- and RF-connections is disadvantageous for these transitions. The transitions to dielectric waveguides are based on different principles. In [6] the wave couples from a half-mode substrate integrated waveguide (SIW) into the dielectric waveguide with a transition loss of $4.8 \mathrm{~dB}$. Other transitions are based on resonant structures like a dielectric resonator [9] (IL: 2.15 dB) or patch antennas [10] (IL: $3.1 \mathrm{~dB}$ ) to excite a propagating wave in the dielectric waveguide. Compared to the transitions to the rectangular waveguide, the insertion loss is increased but

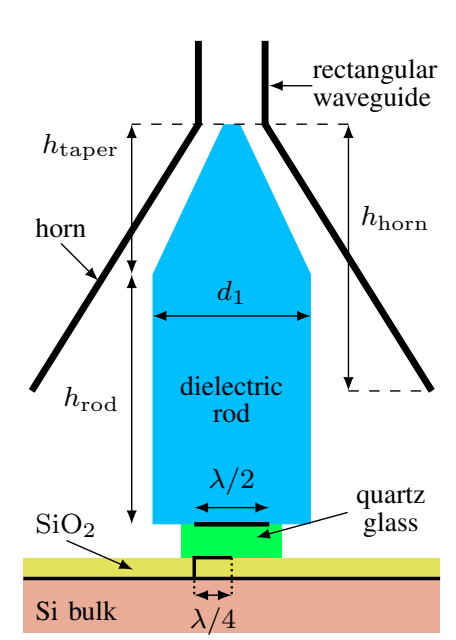

(a)

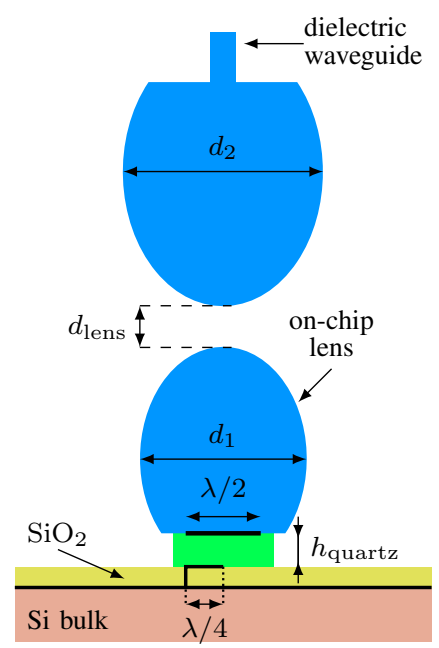

(b)
Fig. 1. Schematic cross-section of the transition from MMIC to rectangular waveguide (a) and to dielectric waveguide (b).

the packaging is less complex. The MMIC can be soldered onto the printed circuit board (PCB), making it simple to connect supply voltages and RF signals.

For all transitions the waveguides are in contact with the MMIC. If the waveguide is exposed to mechanical stress, the MMIC performance can be impaired or the MMIC can be destroyed. Especially for radar systems being used in harsh environments [2], the mechanical decoupling of waveguide and MMIC is therefore an additional design requirement.

This paper presents two mechanically decoupled transitions from MMIC to rectangular and to dielectric waveguide at $160 \mathrm{GHz}$. Both transitions were designed and measured for a back end of line (BEOL) MMIC whose manufacturing process is comparable to the $130-\mathrm{nm}$ SiGe BiCMOS process from IHP microelectronics. In the following section the transition principle for both approaches is explained in detail. In Section III the measurement results of the realized transitions are presented.

\section{TRAnsition PRINCIPLE}

The proposed transition principles from MMIC to rectangular waveguide (Fig. 1a) and to dielectric waveguide (Fig. 1b) can be split into different parts. The first part of both transitions is the signal decoupling of the MMIC with an on-chip antenna, which is the same for both transitions. 


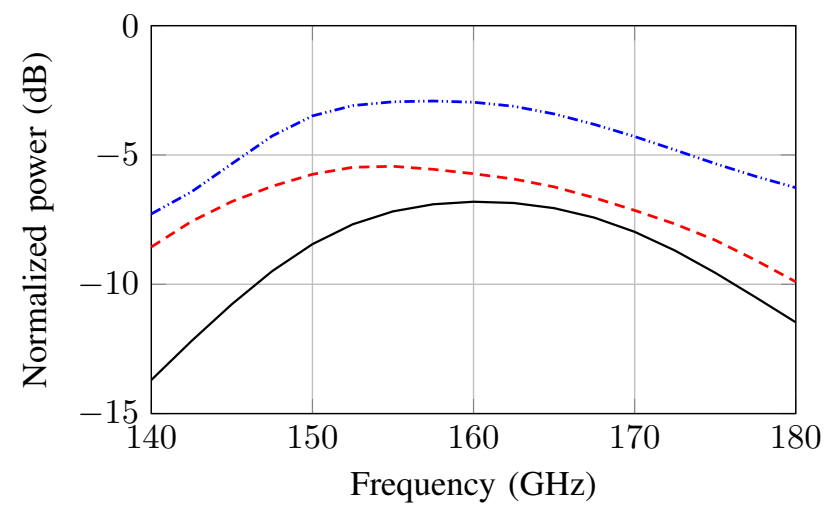

Fig. 2. Simulated radiated power for a quartz glass based antenna ( - ), a quartz glass based antenna with a dielectric rod above (----), and a dielectric lens above (-...-) in the angular range $|\theta|<30^{\circ}$ normalized to the total radiated power.

A focusing element, concentrating the signal power in the desired direction, is placed on the antenna. Since the transitions are mechanically decoupled, the signal propagates for a short distance in free space and is received by a second antenna, which is connected to the respective waveguide. The individual components were investigated by full-wave simulations and are described in the following sections in detail.

\section{A. On-Chip Antenna}

The radiating element on the MMIC is a $\lambda / 4$-patch on the top layer of the $\mathrm{SiO}_{2}$ substrate $\left(\varepsilon_{r, \mathrm{SiO}_{2}}=4, \tan \delta=0.02\right)$ [11]. The $\lambda / 4$-patch is short-circuited to ground in order to couple the field efficiently to a $\lambda / 2$-patch on a quartz glass carrier placed above it [12]. The length of the radiating patch determines the center frequency of the antenna. The coupling from MMIC to patch is affected by the quartz glass thickness and the $\lambda / 4$-patch dimensions.

Due to the stacked design the antenna area on the MMIC is only $170 \mu \mathrm{m} \times 220 \mu \mathrm{m}=0.01 \lambda_{0}^{2}$. The antenna has a simulated bandwidth of $27 \mathrm{GHz}$ and a $3 \mathrm{~dB}$ beamwidth of approximately $75^{\circ}$. In a mechanically decoupled transition, the radiated signal is received by a second antenna. Therefore, the beam of the on-chip antenna should be highly focused on the receiving antenna.

For the presented on-chip antenna, the simulated radiated power in the angular range for $|\theta|<30^{\circ}$ is $-6.8 \mathrm{~dB}$ as shown in Fig. 2. The simulated radiated power is normalized to the total input power, hence the material losses on the MMIC of $2 \mathrm{~dB}$ are already included.

\section{B. Beam Focusing and Receive Antenna}

To decrease the insertion loss of the transition, additional focusing antenna parts are added to the on-chip antenna. The two different transition concepts are explained in the following sections.

\section{1) Dielectric Rod with Horn according to Fig. Ia}

With a dielectric rod antenna the gain of the on-chip antenna can be increased. The fundamental mode in the

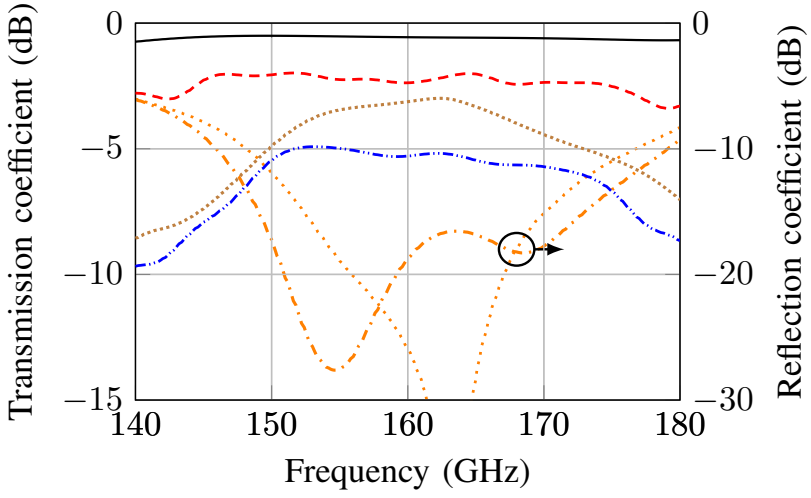

Fig. 3. Simulated insertion loss $\left|s_{21}\right|$ for the transition from dielectric rod to horn $(-)$, lens to lens (----), MMIC to rectangular waveguide $(\cdots \cdots \cdots \cdot \ldots)$, and MMIC to dielectric waveguide (-.-.-) on the left $y$-axis. Reflection coefficient $\left|s_{11}\right|$ from MMIC to rectangular waveguide (......) and from MMIC to dielectric waveguide $(-\cdot-\cdot-)$ on the right $y$-axis.

Table 1. Dimensions for both transitions from Fig. 1.

\begin{tabular}{c|c|c|c|c|c|c}
$\begin{array}{c}\text { Trans. } \\
\text { principle }\end{array}$ & $\begin{array}{c}h_{\text {quartz }} \\
\text { in } \mu \mathrm{m}\end{array}$ & $\begin{array}{c}w_{\text {patch }} \\
\text { in } \mu \mathrm{m}\end{array}$ & $\begin{array}{c}l_{\text {patch }} \\
\text { in } \mu \mathrm{m}\end{array}$ & $\begin{array}{c}d_{1} \\
\text { in } \mathrm{mm}\end{array}$ & $\begin{array}{c}h_{\text {rod }} \\
\text { in } \mathrm{mm}\end{array}$ & $\begin{array}{c}d_{2} \\
\text { in } \mathrm{mm}\end{array}$ \\
\hline \hline Fig. 1a & 127 & 280 & 400 & 1.2 & 3.7 & $\mathrm{x}$ \\
\hline Fig. 1b & 100 & 265 & 400 & 4 & $\mathrm{x}$ & 6
\end{tabular}

dielectric waveguide, the $\mathrm{HE}_{11}$ mode, is excited by the $\lambda / 2$-patch. By tapering the end of the waveguide, the normalized simulated radiated power in the angular range for $|\theta|<30^{\circ}$ is increased to $-5.4 \mathrm{~dB}$.

A horn antenna is used as receive element [11]. The reverse taper of horn and rod allows both shapes to approach each other and, therefore, to minimize the free-space distance in between. As a result, the radiated power can be received by the horn at larger angles. A minimum insertion loss is achieved by equal tapering angles of rod and horn.

The simulated insertion loss for the non-resonant transition from the acryl glass rod to the horn is about $0.5 \mathrm{~dB}$ as shown in Fig. 3. The dielectric waveguide has a taper length $h_{\text {taper }}$ of $1.5 \mathrm{~mm}$ and a diameter $d_{1}$ of $1.2 \mathrm{~mm}$. The horn exhibits a taper angle of $19^{\circ}$ in the E-plane and $28^{\circ}$ in the H-plane. With a length of $h_{\text {horn }}=4.13 \mathrm{~mm}$ the horn covers the dielectric waveguide with its length of $h_{\text {rod }}=3.7 \mathrm{~mm}$ nearly completely.

The simulated insertion loss from the MMIC to the rectangular waveguide is about $3 \mathrm{~dB}$ (cf. Fig. 3). Since the horn covers a large area, the loss is decreased compared to the previously mentioned $5.4 \mathrm{~dB}$, and the radiation loss is only $0.6 \mathrm{~dB}$. The simulated reflection coefficient is below $-10 \mathrm{~dB}$ from $148 \mathrm{GHz}$ to $178 \mathrm{GHz}$. The dimensions for this transition are shown in Table 1.

\section{2) Dielectric Lens Antennas according to Fig. $1 b$}

Another possibility to increase the antenna gain is the use of an elliptical lens [13], which is positioned on the quartz glass. The lens can be designed according to [14] and is fed in the focal point. Thus, the normalized radiated power in the angular range for $|\theta|<30^{\circ}$ is further increased to $-2.9 \mathrm{~dB}$ 
(Fig. 2). The reflection coefficient of the lens antenna is below $-10 \mathrm{~dB}$ from $146 \mathrm{GHz}$ to $179 \mathrm{GHz}$ as shown in Fig. 3 .

A horn antenna as used in the previous section is too small to cover the lens area. By widening the horn the free-space distance increases. Therefore, a second dielectric elliptical lens is used instead as receive antenna in a distance $d_{\text {lens }}$ of $5 \mathrm{~mm}$ to the on-chip lens. Due to the small lens dimensions, the wave is not completely concentrated in the focal point. Therefore, the ellipsoid is extended by $550 \mu \mathrm{m}$ and connected to a dielectric waveguide (cross-section: $800 \mu \mathrm{m} \times 1295 \mu \mathrm{m}$ ) as described in [14]. By tapering the cross-section of the dielectric waveguide, the dimensions are reduced to $648 \mu \mathrm{m} \times 1295 \mu \mathrm{m}$. The further dimensions are specified in Table 1.

For the transition from lens to lens, the radiation loss for the free-space distance is about $2 \mathrm{~dB}$ as shown in Fig. 3. As a result, the insertion loss from MMIC to dielectric waveguide is approximately $4.7 \mathrm{~dB}$. Compared to the transition explained in Section II-B1 the loss is increased by $2 \mathrm{~dB}$. Despite the higher loss, the design is more robust with respect to fabrication tolerances. Furthermore, a transition to dielectric waveguides is desirable in the mm-wave range, since rectangular waveguides are more lossy [5].

\section{Transition Assembly and Measurement}

For the characterization of the transitions, a BEOL MMIC with a back-end height of $10 \mu \mathrm{m}$ between top and bottom layer was fabricated. The $\lambda / 4$-patch is fed by a microstrip line. Since the mounting plates of the transitions have an edge length of $50 \mathrm{~mm}$, the BEOL MMIC has a length of $36 \mathrm{~mm}$ to place the probe tips. The insertion loss of the transition is determined by de-embedding the measured losses of the microstrip line $(35 \mathrm{~dB} \hat{=} 1 \mathrm{~dB} / \mathrm{mm})$ and the probe tips $(3.5 \mathrm{~dB})$. The measurement of the reflection coefficient with the probes is not possible due to the high substrate losses.

The BEOL MMIC was glued on a carrier substrate. The quartz glass, which is metallized with a $2 \mu \mathrm{m}$ thick aluminum layer in a cleanroom process, was glued on the MMIC above the $\lambda / 4$-coupler with epoxy adhesive. On top of the quartz glass the focusing elements, the dielectric rod respectively the lens were also fixed with epoxy adhesive.

\section{A. Transition MMIC to Rectangular Waveguide}

For the assembly of the rectangular waveguide above the MMIC a mounting plate was fixed on the carrier substrate with screws and spacers. For an exact alignment of the horn above the rod a transparent plastic block with a cross-hair is placed in a cavity on the mounting plate. Afterwards, the block is removed and the horn with a rectangular waveguide flange in split-block design is placed in the cavity. The realized transition from MMIC to rectangular waveguide is shown in Fig. 4.

The measured transmission coefficient with a maximum of $-3.1 \mathrm{~dB}$ at $147.1 \mathrm{GHz}$ is shown in Fig. 5. Compared to the simulated transmission coefficient in Fig. 3, the insertion loss is shifted by $12 \mathrm{GHz}$ to lower frequencies. This results from incorrectly assumed material parameters and the neglected

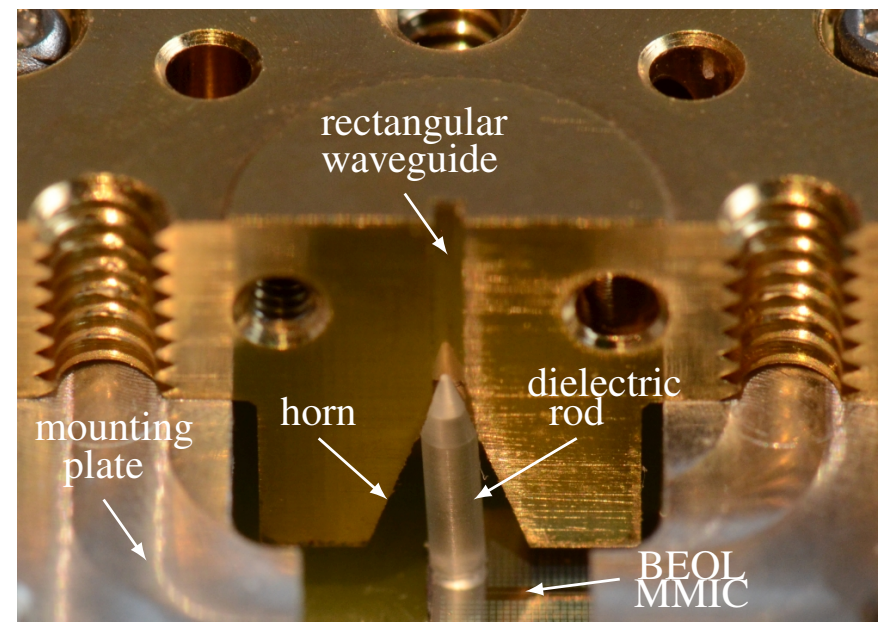

Fig. 4. Realized transition from MMIC to rectangular waveguide (cut in the middle to take photo).

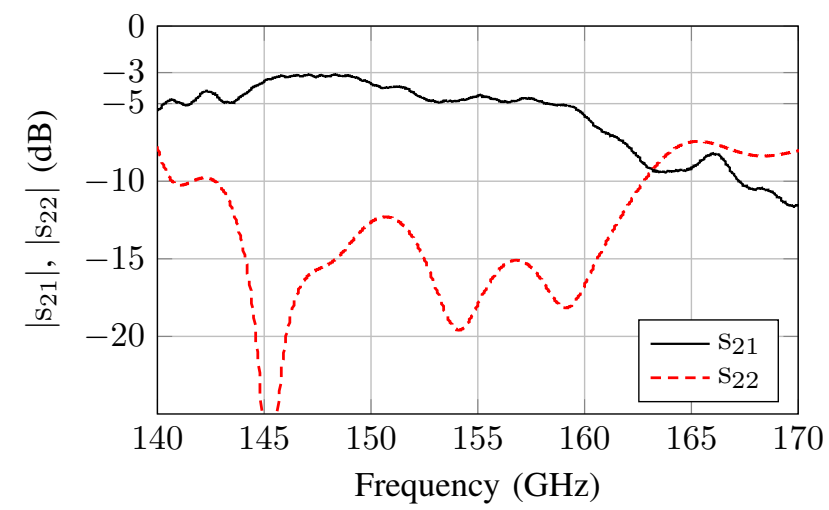

Fig. 5. Measured S-parameters for the transition from MMIC to rectangular waveguide.

epoxy in the simulation. From $140.7 \mathrm{GHz}$ to $162.8 \mathrm{GHz}$ the measured reflection coefficient at the rectangular waveguide interface is below $-10 \mathrm{~dB}$. In this frequency range also the transmission declines by approximately $3 \mathrm{~dB}$ compared to the maximum value.

\section{B. Transition MMIC to Dielectric Waveguide}

In order to mount the receive lens in a defined distance to the on-chip lens, a suitable holding for the lens is required. Therefore, lens and mounting plate were made of one HDPE block. The material around the lens was milled out, and only two narrow bars position the lens as shown in Fig. 6a. A hole is drilled in the lens to plug in the dielectric waveguide. Since bond wires should be connected to the MMIC, the elliptical lens shape was modified by chamfering the bottom side (cf. Fig. 6b). The lens characteristics are not changed since the wave is still focused in the focal point. For setups with a complete system on chip, it is advisable to use a packaged MMIC [15] as no bond wires can be damaged. The sensitive alignment of the lens is also simplified by a lens cavity integrated in the package, because tilting of $2^{\circ}$ already increases the insertion loss by $0.5 \mathrm{~dB}$. 


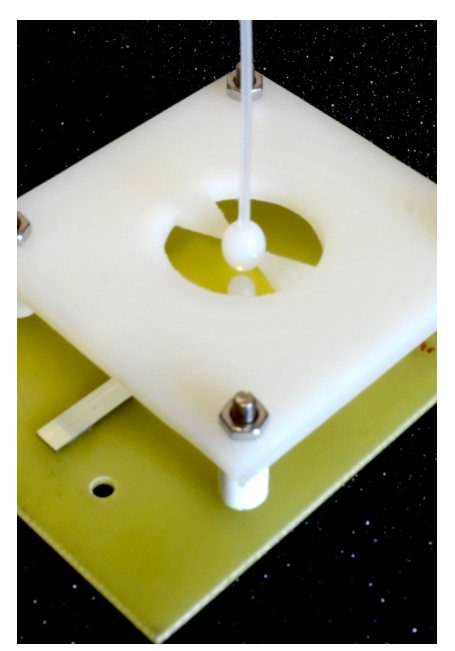

(a) Top view.

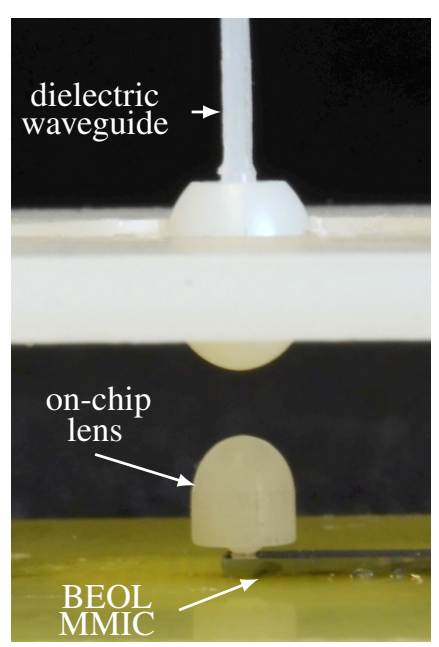

(b) Side view
Fig. 6. Realized transition from MMIC to dielectric waveguide with top view (a) and side view (b).

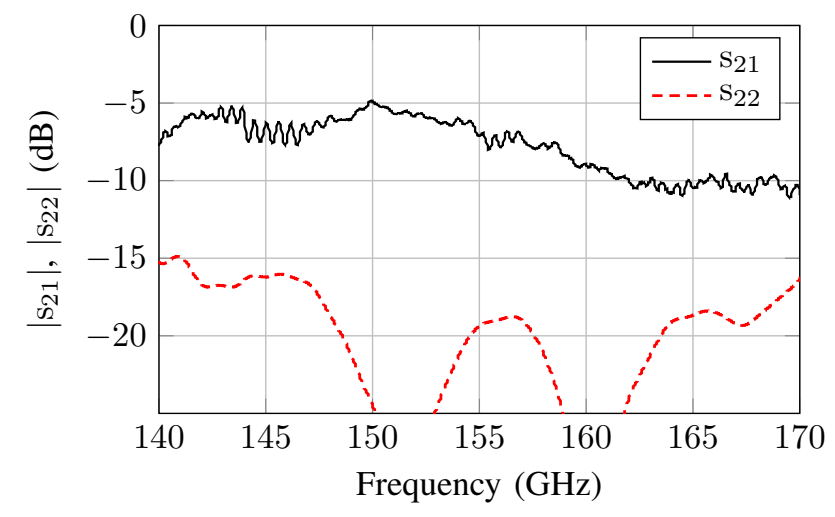

Fig. 7. Measured S-parameters for the transition from MMIC to dielectric waveguide.

The maximum of the transmission coefficient is $-4.9 \mathrm{~dB}$ at a frequency of $150 \mathrm{GHz}$ as shown in Fig. 7. In the frequency range from $140 \mathrm{GHz}$ to $157.5 \mathrm{GHz}$ the transmission declines by $3 \mathrm{~dB}$ compared to the maximum measured value. The measured S-parameter curves are again shifted by approximately $12 \mathrm{GHz}$ to lower frequencies for the same reasons as mentioned in the previous section. The reflection coefficient at the dielectric waveguide is in the complete measured frequency range below $-14 \mathrm{~dB}$.

\section{CONCLUSION}

In this paper, two transitions from MMIC to rectangular waveguide and to dielectric waveguide are presented. The transitions in the G-Band are mechanically decoupled to avoid mechanical stress on the MMIC.

Both transitions are based on a quartz glass carrier with a resonant $\lambda / 2$-patch, which is excited by the MMIC. A focusing element, either a dielectric rod or a dielectric lens on top of the quartz glass increases the directivity of the on-chip antenna. Different high gain antennas (horn and dielectric lens) are used as receiving elements in a minimum distance. S-parameter measurements were done with a BEOL MMIC. The minimum measured insertion loss from MMIC to rectangular waveguide is $3.1 \mathrm{~dB}$ and the minimum insertion loss from MMIC to dielectric waveguide is $4.9 \mathrm{~dB}$.

Compared to state-of-the-art designs [6]-[10], the insertion loss is increased due to the mechanical decoupling of waveguide and MMIC. In contrast to most of the compared designs, a standard IC process is used for the transitions with a low chip area consumption. In addition, great importance was attached to a simple and cheap implementation on PCBs.

\section{ACKNOWLEDGMENT}

This work was supported by the Ministry for Science, Research and Arts Baden-Württemberg within the project ZAFH MikroSens. Furthermore, the authors would like to thank Stefan Jenisch, Markus Mohr, Rudolf Rösch, and Katrin Schiller for the technical support.

\section{REFERENCES}

[1] M. Hitzler et al., "Ultracompact 160-GHz FMCW Radar MMIC With Fully Integrated Offset Synthesizer," IEEE Trans. Microw. Theory Techn., vol. 5, no. 65, pp. 1682-1691, May 2017.

[2] S. Kueppers et al., "A Compact $120 \mathrm{GHz}$ SiGe:C Based $2 \times 8$ FMCW MIMO Radar Sensor for Robot Navigation in Low Visibility Environments," in European Radar Conference (EURAD), Oct. 2017, pp. 122-125.

[3] N. Sarmah et al., "A Wideband Fully Integrated SiGe Chipset for High Data Rate Communication at $240 \mathrm{GHz}$," in 11th European Microwave Integrated Circuits Conference (EuMIC), Oct. 2016, pp. 181-184.

[4] N. V. Thienen et al., "A Multi-Gigabit CPFSK Polymer Microwave Fiber Communication Link in $40 \mathrm{~nm}$ CMOS," IEEE J. Solid-State Circuits, vol. 51, no. 8, pp. 1952-1958, Aug. 2016.

[5] M. Geiger et al., "A 160-GHz Radar With Flexible Antenna Used as a Sniffer Probe," IEEE Sensors J., vol. 17, no. 16, pp. 5104-5111, Aug. 2017.

[6] J. W. Holloway et al., "A Fully Integrated Broadband Sub-mmWave Chip-to-Chip Interconnect," IEEE Trans. Microw. Theory Techn., vol. 65, no. 7, pp. 2373-2386, Jul. 2017.

[7] V. Vassilev et al., "Integrated Front-ends up to $200 \mathrm{GHz}$," in IEEE MTT-S International Microwave Workshop Series on Millimeter Wave Integration Technologies, Sep. 2011, pp. 57-60.

[8] K. M. K. H. Leong et al., "A $340-380 \mathrm{GHz}$ Integrated CB-CPW-to-Waveguide Transition for Sub Millimeter-Wave MMIC Packaging," IEEE Microw. Wireless Compon. Lett., vol. 19, no. 6, pp. 413-415, Jun. 2009.

[9] U. Dey and J. Hesselbarth, "Building Blocks for a Millimeter-Wave Multiport Multicast Chip-to-Chip Interconnect Based on Dielectric Waveguides," IEEE Trans. Microw. Theory Techn., vol. 66, no. 12, pp. 5508-5520, Dec. 2018.

[10] B. Yu et al., "Ortho-Mode Sub-THz Interconnect Channel for Planar Chip-to-Chip Communications," IEEE Trans. Microw. Theory Techn., vol. 66, no. 4, pp. 1864-1873, Apr. 2018.

[11] M. Hitzler et al., "MMIC-to-Waveguide Transition at $160 \mathrm{GHz}$ with Galvanic Isolation," in IEEE MTT-S International Microwave Symposium (IMS), May 2016, pp. 1-4.

[12] J. Hasch et al., "77 GHz Radar Transceiver with Dual Integrated Antenna Elements," in German Microwave Conference Digest of Papers, Mar. 2010, pp. 280-283.

[13] D. F. Filipovic et al., "Double-Slot Antennas on Extended Hemispherical and Elliptical Silicon Dielectric Lenses," IEEE Trans. Microw. Theory Techn., vol. 41, no. 10, pp. 1738-1749, Oct. 1993.

[14] M. Geiger et al., "A Dielectric Lens Antenna Fed by a Flexible Dielectric Waveguide At $160 \mathrm{GHz}$," in 11th European Conference on Antennas and Propagation (EuCAP), Mar. 2017, pp. 3380-3383.

[15] M. Hitzler et al., "Radiation Pattern Optimization for QFN Packages With On-Chip Antennas at $160 \mathrm{GHz}$," IEEE Trans. Antennas Propag., vol. 66, no. 9, pp. 4552-4562, Sep. 2018. 